\title{
The Use of Brentuximab Vedotin with or without Subsequent Allogeneic Stem Cell Transplantation in Selected Patient Groups
}

\author{
Ulrich Jager, ${ }^{1}$ Felix Keil ${ }^{2}$ and Martin Hutchings ${ }^{3}$
}

1. Professor of Hematology, Medical University of Vienna, Vienna, Austria; Head of the Clinical Department of Hematology and Hemostasis, Vienna General Hospital, Vienna, Austria; 2. Professor of Hematology, Hanusch Hospital, Vienna, Austria; 3. Clinical Oncologist, Department of Hematology, Rigshospitalet, Copenhagen, Denmark

\begin{abstract}
While combined chemotherapy regimens can be effective in classical Hodgkin's lymphoma (CHL) and systemic anaplastic large cell lymphoma (SALCL), around a third of patients are unresponsive or relapse within a few years of treatment. Allogeneic stem cell transplantation (alloSCT) may achieve long-term disease control in patients with chemoresistant disease, but many patients are unable to undergo allo-SCT. Brentuximab vedotin (BV) is a novel antibody-drug conjugate targeting CD30. Recent data have suggested that this treatment may be beneficial in patients who have had an unsatisfactory response to at least two prior treatments and are not eligible for allo-SCT. In addition, BV may be used to reduce tumour burden, allowing patients to subsequently progress to allo-SCT. This article discusses case studies of treatment-refractory patients with $\mathrm{CHL}$ and SALCL, for whom BV has provided durable complete responses (CRs).
\end{abstract}

\section{Keywords}

Classical Hodgkin's lymphoma (CHL), systemic anaplastic large cell lymphoma (SALCL), brentuximab vedotin

\begin{abstract}
Disclosure: Ulrich Jager has received honoraria and participated in Advisory Boards for Takeda. Felix Keil has nothing to disclose is relation to this article. Martin Hutchings has participated in Advisory Boards for Takeda, Celgene, Genentech and Janssen, and has been a consultant to Takeda and Genentech. Acknowledgements: Medical writing assistance was provided by Katrina Mountfort at Touch Medical Media and funded by Takeda.

Compliance with Ethics Guidelines: All procedures were followed in accordance with the responsible committee on human experimentation and with the Helsinki Declaration of 1975 and subsequent revisions. Informed consent was received from the patients involved in these case studies.

open Access: This article is published under the Creative Commons Attribution Noncommercial License, which permits any non-commercial use, distribution, adaptation and reproduction provided the original author(s) and source are given appropriate credit.

Received: 25 May 2015 Accepted: 26 October 2015 Citation: European Oncology \& Haematology, 2015;11(2):130-3 DOl: http://doi.org/10.17925/EOH.2015.11.02.130 Correspondence: Ulrich Jager, Medical University of Vienna, Vienna, Spitalgasse 23, 1090 Wien, Austria. E: ulrich.jaeger@meduniwien.ac.at; Martin Hutchings, Department of Haematology, Rigshospitalet, 9 Blegdamsvej, 2100, Copenhagen, Denmark. E: martin.hutchings@gmail.com
\end{abstract}

Support: The publication of this article was supported by Takeda, who were given the opportunity to review the article for scientific accuracy before submission. Any resulting changes were made at the author's discretion.

Chemotherapy achieves excellent response rates and long progressionfree survival (PFS) in classical Hodgkin's lymphoma (CHL) and systemic anaplastic large cell Iymphoma (SALCL). ${ }^{1}$ However, up to $30 \%$ of patients with advanced $\mathrm{HL}$ do not achieve remission or relapse within a few years of initial treatment.2 Salvage chemotherapy followed by high-dose chemotherapy and autologous stem cell transplantation $(\mathrm{HD}+\mathrm{ASCT})$ is the standard of care for patients with chemosensitive refractory or relapsed $\mathrm{CHL} .{ }^{1}$ However, the prognosis is poor for patients who relapse following $\mathrm{HD}+\mathrm{ASCT}{ }^{3}$ Independent risk factors for overall survival (OS) include early relapse ( $<6$ months) after ASCT, stage IV, bulky disease, poor performance status (PS) and age $\geq 50$ years at relapse. ${ }^{4}$ For patients with SALCL, the benefits of SCT are less clear, and patients not achieving a complete response (CR) or at least a good partial response (PR) before HD+ASCT usually have a poor prognosis. Moreover, patients that relapse after HD+ASCT have few effective treatment options.

In patients with chemo-refractory disease who are otherwise clinically fit, allogeneic stem cell transplantation (allo-SCT) has been shown to have a curative potential as a result of the underlying graft-versus-
Iymphoma (GVL) effect. However, many patients are unable to undergo allo-SCT, and treatment with brentuximab vedotin (BV) provides an alternative for these patients. On the basis of a number of pivotal clinical trial data, ${ }^{5-8} \mathrm{BV}$ was granted accelerated approval by the US Food and Drug Administration (FDA) and conditional approval by the European Medicines Agency (EMA) for the treatment of patients with $\mathrm{CHL}$ after failure of auto-SCT or after failure of at least two prior chemotherapy regimens in patients who are not auto-SCT candidates, and for the treatment of patients with SALCL after failure of at least one prior combined chemotherapy regimen. This article aims, through a series of case studies, to demonstrate the use of BV in selected patient groups with relapsed/refractory $\mathrm{CHL}$ and SALCL.

\section{The Use of Allogeneic Stem Cell Transplantation in Relapsed/Refractory Classical Hodgkin's Lymphoma and Systemic Anaplastic Large Cell Lymphoma}

The role of allo-SCT in CHL is not well established and is considered controversial. ${ }^{9-13}$ The treatment is associated with significant toxicity and results in few long-term disease-free survivors. Reduced-intensity 
conditioning (RIC) has lowered the treatment-related mortality; however it confers an increased risk of early relapse, with disease relapse and progression reported in more than half of patients. ${ }^{14-16}$ In addition, there are limited treatment options for patients who relapse after allo-SCT, in part due to the challenge of giving systemic antineoplastic therapy to the graft bone marrow cells. ${ }^{17}$ An expert panel has recommended that allo-SCT may be used in a tandem strategy after HD+ASCT, provided the patient is sensitive to salvage chemotherapy and does not display disease progression between the two transplants. allo-SCT may also be considered in patients relapsing after an ASCT, provided the patient is sensitive to salvage chemotherapy. ${ }^{10,18}$

\section{Factors that Prevent Patients from Proceeding to Stem Cell Transplantation}

A significant proportion of patients are not eligible for SCT, including those who do not either achieve sufficient clinical response to proceed to transplantation, or harbour a chemosensitive disease. In addition, elderly patients, or those with comorbidities, may be unable to tolerate intensive therapy.

There are three distinct patient subgroups for which BV may be particularly beneficial:

- Patients with CHL who relapse after HD+ASCT and therefore are potential candidates for allo-SCT. Only relatively young $(<70)$, fit patients are eligible for the latter.

- Patients with $\mathrm{CHL}$ who have had an unsatisfactory response to at least two prior treatments and are not eligible for SCT.

- Any patient with SALCL who has failed the standard CHOP regimen (cyclophosphamide, doxorubicin, vincristine and prednisolone).

\section{Brentuximab Vedotin as a Bridge to Stem Cell Transplantation in Clinical Studies}

Several studies have investigated the use of BV as a bridge to transplantation in patients who might not otherwise fulfil the criteria for allo-SCT. A retrospective study of 14 patients with refractory or relapsed $\mathrm{CHL}$ who had not received prior $\mathrm{HD}+\mathrm{ASCT}$ due to refractory disease $(n=9)$, comorbidity $(n=4)$ and unknown reason $(n=1)$, found that BV resulted in an overall response rate (ORR) of $71 \%$ with CR in $36 \%$, and allowed consolidating HD+ASCT to be performed in four patients and allo-SCT in one patient. ${ }^{19} \mathrm{BV}$ has also been investigated as a means of reducing tumour burden to facilitate consolidative allo-SCT, improving post-transplantation disease-free survival. Fifteen patients who participated in the pivotal phase II studies of BV received consolidative allo-SCT following BV treatment. The 2-year PFS of these patients was estimated to be $66 \%$ and the median PFS had not yet been reached at the time of reporting. Eleven patients were alive and the estimated 2-year OS rate was $80 \% .^{20}$

In a retrospective analysis, the outcomes of $23 \mathrm{cHL}$ patients who underwent RIC allo-SCT with no prior BV exposure were compared with those of 21 additional $\mathrm{CHL}$ patients who had received BV prior to RIC allo-SCT. The patients who received BV prior to RIC allo-SCT showed better haematopoietic cell transplantation comorbidity index (HCT-Cl), better remission status at time of transplant and reduced peri-transplant toxicity. ${ }^{21}$ In another study of patients who underwent allo-SCT ( $\mathrm{n}=27$ ), $52 \%$ received BV prior to allo-SCT, and in seven patients, it was the last therapy prior to allo-SCT. In these seven patients the CR rate was $100 \%$, indicating that a BV-based transplant strategy is useful in this high-risk patient group.22

\section{Figure 1: Case 1}
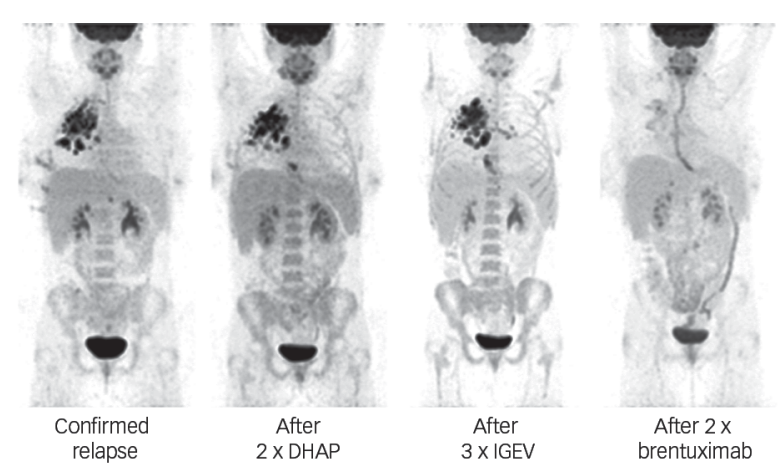

\section{Brentuximab Vedotin as a Bridge to Stem Cell Transplantation in Real Life Clinical Practice}

In order for BV to be used as an alternative to allo-SCT in clinical practice, several practical considerations must be addressed. The optimal timing of allo-SCT after BV response in this setting remains a challenge. In addition, it is not known who would benefit from allo-SCT soon after reaching remission versus sustained treatment with BV. In an Italian study $(n=65)$, best ORR were obtained after three to four cycles..$^{23} \mathrm{~A}$ recent Turkish study $(n=58)$, found that BV provided a bridge to transplantation in approximately one-quarter of the patients. However, responses were not always sustained during the course of treatment suggesting that transplantation should be implemented early during treatment and not as late as after up to 16 treatment cycles. ${ }^{24}$

\section{Case Studies}

\section{Case 1 - Classical Hodgkin's Lymphoma}

A 20-year-old female was diagnosed with primary progressive $\mathrm{CHL}$ in summer 2011. The disease was stage IIEA with large mediastinal bulk. First-line treatment was four cycles of ABVD (doxorubicin, bleomycin, vinblastine and dacarbazine) followed by radiotherapy. In June 2012, relapse was confirmed. The patient received two cycles of DHAP (dexamethasone, cytarabine and cisplatin) resulting in no response and subsequently three cycles of IGEV (ifosfamide, gemcitabine, vinorelbine and prednisone), also without any clinical benefit. However, after two cycles of BV, CR was observed (see Figure 1). The patient received eight cycles of BV until spring 2013. In June 2013 the patient received HD+ASCT and is still in CR.

\section{Case 2 - Hodgkin's Lymphoma (Mixed Cellularity)}

A 65-year-old was admitted in December 2014 with CHL, mixed type, since July 2010, stage IVB. One cycle of escalated BEACOPP (bleomycin, etoposide, doxorubicin, cyclophosphamide, vincristine, procarbazine and prednisolone) led to massive toxicity. However, following six cycles of BEACOPP, partial remission was seen. In August 2011, progression of the underlying illness was seen, with suspected liver and spleen infiltration, generalised lymph node involvement, mediastinal, hilar, retroperitoneal, iliac and mesenterial. However there was no evidence that the underlying disease had attacked the bone. The patient underwent stem cell apheresis in February 2012, followed by HD+ASCT in March 2012. However, disease progression was observed in November 2012 positron emission tomography (PET)-positive mediastinal, abdominal and osseous foci. The patient received 10 cycles of BV until June 2013. Progression was noted in December 2013 but at restaging in February 2014, a distinct improvement in findings was noted. The patient was subsequently treated with gemcitabine and everolimus. The patient is alive and well and still under treatment with gemcitabine and everolimus. 


\section{Case 3 - Anaplastic Large Cell Lymphoma}

A 36-year-old male who had been HIV-positive since 2009 presented in May 2012 with enlarged cervical lymph nodes. He had been taking highly active antiretroviral therapy (HAART) since April 2012 and had no signs of a deficient immune system. Following an excisional biopsy, bone marrow biopsy (BMB) and a computed tomography (CT) scan, a diagnosis of anaplastic lymphoma kinase negative (ALK-) ALCL stage IIB with bulky mediastinal disease was made. First-line treatment in August 2012 was six cycles of CHOEP14 (cyclophosphamide, doxorubicin, vincristine, etoposide and prednisone). In September 2012 the patient's stem cells were successfully harvested. A CT scan showed a PR. In October 2012, the patient underwent HD BEAM (carmustine, etoposide, cytarabine and melphalan) and ASCT. Subsequent CT showed a CR.

The first relapse occurred in June 2013, diagnosed after 2 weeks of unexplained fever. CT showed a progressive mediastinal tumour, and no visible disease outside the mediastinum. A thoracoscopic biopsy revealed ALK- ALCL. A new PET/CT scan was performed to exclude localised disease. This revealed involvement of the mediastinum, supraclavicular lymph nodes, retroperitoneum and a pericardial effusion. A registry search was performed to find a stem cell donor, since there was no available sibling, and second-line treatment was initiated. The patient started BV in June 2013. In August 2013, a PET/CT scan showed a PR after four cycles. After six cycles, in October 2013, a PET/CT scan showed CR. In November, the patient learned that there was no matching stem cell registry donor; however, in January 2014, two matching umbilical cord blood (UCB) portions were found, therefore the patient was accepted for allo-SCT. In March 2013 he received the last of 13 BV cycles. The only adverse events reported were grade I fatigue and one febrile episode. In March 2014, a PET/CT scan and BMB revealed that the patient was still in CR. In March, the patient underwent UCB non-myeloablative SCT. He was discharged in May and was well, with no signs of graft-versus-host disease (GvHD).

\section{Case 4 - Anaplastic Large Cell Lymphoma}

A 33-year-old male first presented in July 2012 following 2 weeks of fever and lymph node enlargement. Other symptoms included dyspnoea, chest pain, elevated troponin T (TnT) and left ventricular ejection fraction (LVEF) 35-40 \%. In August 2012, a PET/CT found showed sign of disease in all nodal regions, PET-positive subcutaneous lesions, splenic disease. An echocardiogram showed perimyocarditis but no cardiac lymphoma infiltration. Following a needle biopsy from an inguinal lymph node, a diagnosis of ALK+ ALCL was made and despite a negative BMB the patient had stage IVB disease. From August 2012 the patient was given six cycles of CHOEP14. No HD+ASCT was planned since the patient had ALK+ disease. The chemotherapy was generally well tolerated with mild side effects with almost immediate and full resolution of diseaserelated symptoms. In October 2012 a CR was confirmed on PET/CT after four cycles. In November, the CR was maintained on PET/CT 3 weeks after the sixth cycle. However, following recurrent fever and malaise, the patient was readmitted to the clinic in February 2012. PET and CT scans showed progressive, PET-positive disease in most nodal regions. A subsequent axillary biopsy showed ALK+ ALCL. In February 2013, the patient received four cycles of GDP (gemcitabine, dexamethasone and cisplatin). A CR was confirmed on PET/CT after two cycles. A successful $\mathrm{SC}$ harvest was made after the fourth cycle, with relatively severe side effects including febrile neutropenia, abdominal pain, diarrhoea and deep vein thrombosis (DVT). In May, the patient underwent HD BEAM and ASCT. He suffered severe side effects including mucositis and pulmonary embolism but was well after discharge. Two months later, PET/CT showed a CR and the patient returned to work full time.

However, a second relapse occurred in October 2013. The patient reported sudden swelling around the left eye accompanied by respiratory tract infections. A magnetic resonance imaging (MRI) scan of the head showed a large intraorbital tumour. The patient was admitted with progressive symptoms including dyspnoea, hypotension and tachycardia/ventricular tachycardia (VT). A PET/CT scan showed that again all nodal regions were involved, as well as the eye, myocardium, pancreas, kidney and liver, and there was severe pericardial effusion. The patient was treated with pericardiocentesis and subsequent amiodarone. An axillary biopsy led to the diagnosis of ALK + ALCL.

At this point, the patient began third-line treatment with BV. The patient's brother was non-compatible as he was a haematopoietic stem cell (HSC) donor. A PET/CT scan found that a CR had been achieved after three cycles. Seven more cycles of BV were administered between January and May 2014. In March 2014, a compatible HSC registry donor was identified. In April 2014, the patient suffered a Pneumocystic jirovecii infection, which was successfully treated with cotrimoxazole. After this, the patient was accepted for allo-SCT. By May 2014, the patient was still in CR on PET/CT and in June 2014 he underwent non-myeloablative allo-SCT.

\section{Case 5 - Anaplastic Large Cell Lymphoma}

A 47-year-old female was transferred to the intensive care unit (ICU) in January 2012 because of respiratory insufficiency. A diagnosis of ALK+ ALCL stage IV with bone marrow infiltration with CD30 positive lymphoma cells was made. It was unclear whether the diffuse pulmonary problem was due to lymphoma infiltration or pneumonia. The patient received her first cycle of CHOP in January 2012. She recovered quickly after the first CHOP and she received a total of six cycles of CHOP. In March 2012, CR was confirmed, which was maintained at final restaging in May 2012.

In July 2012 the patient had an early relapse in the left mandibular angle. The patient received one cycle of DHAP followed by stem cell harvest as well as one cycle of mini BEAM (she had hearing problems due to platinum therapy). Mini BEAM was initiated on 24 August 2012. Following confirmation of $\mathrm{CR}$, autologous stem cells transplantation was performed in November 2012. A second relapse was diagnosed on 18 December 2012 following a PET scan as well as histology from the right mandibular angle.

The patient received her first cycle of BV in February 2013. She experienced a varicella zoster reactivation at the beginning of cycle three of BV in March 2013, received valaciclovir, but BV was continued. A clinical remission was confirmed on in April 2013 (after the third cycle of BV). After cycle six (June 2013), the patient had suspected Pneumocystis pneumonia, which quickly responded to atovaquone. BV was continued until cycle 12 (September 2013). Shortly after the twelfth infusion the patient developed weakness in both legs (October 2013) and was transferred to the neurological department where a differential diagnosis of brain natriuretic peptide (BNP) or chronic inflammatory demyelinating neuropathy (CIDP) was made. A lumbar puncture confirmed the diagnosis of CIDP. The patient was unable to walk and received high-dose intravenous immunoglobulins.

Symptoms improved gradually with neurological habilitation over several months. In April 2014, the patient was in complete remission from ALCL 
and was last seen in September 2014 in complete clinical remission with some remaining problems in walking. The significance of this case is that the ALK+ CD30+ ALCL responded with complete remission after BV-therapy while this lymphoma was chemotherapy refractory and also refractory to ASCT. The neurological complication was quite unique with only one other case published in the literature. ${ }^{25}$ Nevertheless, the neurological problem finally resolved and the patient remains in continuous $C R$ after 2 years.

\section{Summary and Concluding Remarks}

The clinical cases presented above illustrate the challenges in the treatment of $\mathrm{CHL}$ and SALCL. In both conditions, relapses following chemotherapy frequently occur regardless of initial responses to the treatment. Allo-SCT offers curative potential; however, its use is associated with significant toxicity in all cases. Furthermore, a minority of patients survive allo-SCT in the long term. The CD30-targeted agent BV offers an alternative to allo-SCT and may also be used to reduce tumour burden prior to allo-SCT. The case studies have demonstrated that, while not effective in all cases, BV is capable of inducing durable CRs and provides an additional therapeutic option for patients for whom palliative care has been the only available treatment. These include a case of complete remission in ALK+ CD30+ ALCL. Further clinical studies are required to determine whether treatment with $\mathrm{BV}$ results in improved long-term survival.
1. Schmitz N, Pfistner B, Sextro M, et al., Aggressive conventional chemotherapy compared with high-dose conventional chemotherapy compared with high-dose transplatapy for relapsed chemosensitive sodg-cell drisease: a randomised trial, Lancet, 2002:359:2085-71. 9:2065-71.

2. Kuruvilla J, Standard therapy of advanced Hodgkin lymphoma, Hematology Am Soc Hematol Educ Program, 2009;497-506.

3. Sureda A, Constans M, Iriondo A, et al., Prognostic factors affecting long-term outcome after stem cell transplantation in Hodgkin's lymphoma autografted after a first relapse, Ann Oncol, 2005;16:625-33.

4. Martinez C, Canals C, Sarina B, et al., Identification of prognostic factors predicting outcome in Hodgkin's lymphoma patients relapsing after autologous stem cell transplantation, Ann Oncol, 2013:24:2430-4.

5. Younes A, Gopal AK, Smith SE, et al., Results of a pivotal phase II study of brentuximab vedotin for patients with relapsed or refractony Hodgkin's lymphoma, $J$ Clin Oncol, 2012;30:2183-9.

6. Gopal AK, Chen R, Smith $S$, et al., The three-year follow-up data and characterization of long-term remissions from an ongoing phase 2 study of brentuximab vedotin in patients with relapsed or refractory Hodgkin Lymphoma. Presented at the American Society of Hematology Meeting, New Orleans, 7-10 December 2013; Abstract no 4382.

7. Pro B, Advani R, Brice P, et al., Brentuximab vedotin (SGN-35) in patients with relapsed or refractory systemic anaplastic large-cell lymphoma: results of a phase II study, I Clin Oncol, 2012;30:2190-6.

8. Pro B, Advani R, Brice P, et al., Three year survival results from an ongoing phase 2 study of brentuximab vedotin in patients with relapsed or refractory systemic anaplastic large cell lymphoma. Presented at the American Society of in Abstract no 1809

9. Devetten MP, Hari PN, Carreras 」, et al., Unrelated donor reduced-intensity allogeneic hematopoietic stem cel transplantation for relapsed and refractory Hodgkin Iymphoma, Biol Blood Marrow Transplant, 2009;15:109-17.

10. Peggs KS, Anderlini P, Sureda A, Allogeneic transplantation for Hodgkin Iymphoma, Br J Haematol, 2008:143:468-80.

11. Peggs KS, Kayani I, Edwards N, et al., Donor lymphocyte infusions modulate relapse risk in mixed chimeras and induce durable salvage in relapsed patients after T-celldepleted allogeneic transplantation for Hodgkin's lymphoma, J Clin Oncol, 2011;29:971-8

12. Sureda A, Pereira MI, Dreger P, et al., The role of hematopoietic stem cell transplantation in the treatment of relapsed/refractory Hodgkin's Iymphoma, Curr Opin Oncol, 2012;24:727-32.

13. Robinson SP, Sureda A, Canals C, et al., Reduced intensity conditioning allogeneic stem cell transplantation for Hodgkin's lymphoma: identification of prognostic factors predicting outcome, Haematologica, 2009;94:230-8.

14. Burroughs LM, O'Donnell PV, Sandmaier BM, et al., Comparison of outcomes of HLA-matched related, unrelated, or HLA-haploidentical related hematopoietic cell transplantation following nonmyeloablative conditioning for transplantation following nonmyeloablative conditioning for
relapsed or refractory Hodgkin Iymphoma, Biol Blood Marrow relapsed or refractory Hodgkin

15. Sarina B, Castagna L, Farina L, et al., Allogeneic transplantation improves the overall and progression-fre survival of Hodgkin lymphoma patients relapsing after autologous transplantation: a retrospective study base on the time of HLA typing and donor availability, Blood, 2010;115:3671-7.

16. Ram R, Gooley TA, Maloney DG, et al., Histology and time to progression predict survival for lymphoma recurring after reduced-intensity conditioning and allogeneic hematopoietic cell transplantation, Biol Blood Marrow Transplant. 2011:17:1537-45.

17. Corradini P, Sarina B, Farina L, Allogeneic transplantation for
Hodgkin's lymphoma, Br J Haematol, 2011;152:261-72

18. Van Den Neste $\mathrm{E}$, Casasnovas $\mathrm{O}$, Andre $\mathrm{M}$, et al., Classica Hodgkin's Iymphoma: the Lymphoma Study Association guidelines for relapsed and refractory adult patients eligible for transplant, Haematologica, 2013:98:1185-95.

19. Sasse $S$, Rothe $A$, Goergen $H$, et al., Brentuximab vedotin (SGN-35) in patients with transplant-naive relapsed/refractory Hodgkin Iymphoma, Leuk Lymphoma, 2013;54:2144-8.

20. Illidge $T$, Bouabdallah R, Chen R, et al., Allogeneic transplant following brentuximab vedotin in patients with relapsed or refractory Hodgkin lymphoma and systemic anaplastic large cell lymphoma, Leuk Lymphoma, 2014;1-34.

21. Chen R, Palmer J, Tsai, N-C, et al., Brentuximab vedotin improves $\mathrm{HCT}-\mathrm{Cl}$, CR status, and peri-transplant toxicity in patients with relapsed/refractory Hodgkin lymphoma heading to RIC allo-HCT. Presented at the the American Society of Hematology meeting New Orleans, 7-10 December 2013; Abstract no 3374.

22. Anderlini P, Saliba RM, Ledesma BS, et al., Reduced-intensity conditioning (RIC) and allogeneic stem cell transplantation (allo-SCT) for relapsed/refractory Hodgkin lymphoma (HL) in the brentuximab vedotin era: favorable overall and progression-free survival (OS/PFS) with low transplantrelated mortality (TRM), Clin Adv Hematol Oncol, 2014;12(Suppl. 6):15-7.

23. Zinzani PL, Viviani S, Anastasia A, et al., Brentuximab vedotin in relapsed/refractory Hodgkin's Iymphoma: the Italian experience and results of its use in daily clinical practice outside clinical trials, Haematologica, 2013;98:1232-6.

24. Salihoglu A, Elverdi T, Karadogan I, et al., Brentuximab vedotin for relapsed or refractory Hodgkin lymphoma: experience in Turkey, Ann Hematol, 2015;94:415-20.

25. Pastorelli F, Derenzini E, Plasmati $R$, et al., Severe periphera motor neuropathy in a patient with Hodgkin lymphoma treated with brentuximab vedotin, Leuk Lymphoma, 2013:54:2318-21. 